\section{SITUACIÓN DE LA GESTIÓN DEL RIESGO DE DESASTRES EN EL PATRIMONIO CULTURAL EN COSTA RICA Y SU RELACIÓN CON CENTROAMÉRICA}

\author{
Bernadette Esquivel Morales ${ }^{1, *}$
}

\section{RESUMEN}

Este artículo analiza las acciones que se realizan en la región centroamericana ante las constantes amenazas al patrimonio cultural. La intensidad y frecuencia de las amenazas naturales, aunado a la falta de conservación preventiva y el desinterés de los gobiernos por las edificaciones históricas y formas de vida tradicionales, aumenta su vulnerabilidad. La Gestión del Riesgo de Desastres apenas incorpora el tema del patrimonio cultural en las discusiones mundiales. Los últimos Foros Consultivos de la Política Centroamericana de Gestión Integral de Riesgo de Desastres (PCGIR), realizados en 2013 y 2015, así como el Marco Internacional de Acción de Sendai para la Reducción del Riesgo de Desastre, 2015-2030, establecen como una necesidad el fortalecer y proteger el patrimonio cultural y destinar recursos para lograrlo. A pesar de ello, no se cuenta con los protocolos necesarios para reducir el riesgo de desastres del patrimonio cultural. Se concluye que siendo el patrimonio cultural un derecho humano y su conservación un compromiso mundial, la incorporación de la gestión integral del riesgo en el patrimonio cultural es un deber al que debemos dedicar nuestro mayor esfuerzo.

\section{PALABRAS CLAVE}

Patrimonio cultural, integración, gestión integral de riesgos

\section{ABSTRACT}

This article analyzes the actions carried out in the Central American region in the face of constant threats to cultural heritage. The intensity and frequency of natural hazards, coupled with the lack of preventive conservation and the lack of interest of governments in historical buildings and traditional ways of life, increase their vulnerability. Disaster Risk Management hardly incorporates the theme of cultural heritage into global discussions. The latest Consultative Forums of the Central American Integrated Management of Disaster Risk Management (PCGIR), carried out in 2013 and 2015, and the Sendai Framework for Disaster Risk Reduction 2015-2030, establish as a necessity strengthening and protecting cultural heritage and allocating resources to achieve it. Despite this, there are no protocols necessary to reduce risks of cultural heritage disasters. It is concluded that since cultural heritage is a human right and its conservation a global commitment, the incorporation of integral risk management into cultural heritage is a duty to which we must devote our greatest effort.

\section{KEYWORDS}

Cultural heritage, integration, integrated risk management
1. Centro de Investigación en Identidad Cultural

Latinoamericana, Universidad de Costa Rica, Sede Occidente - San Ramón, Costa Rica.

*Autor de correspondencia: maria.esquivel@ucr.ac.cr

\section{RECIBIDO}

31 de enero de 2017

\section{ACEPTADO}

20 de febrero de 2017

\section{PUBLICADO}

15 de julio de 2017

\section{Formato cita}

Recomendada (APA):

Esquivel Morales, B. (2017). Situación de la Gestión del Riesgo de desastres en el patrimonio cultural en Costa Rica y su relación con Centroamérica. REDER, 1(1), pp.7o-84.

\section{(c) (1) (8)}

Todos los artículos publicados en REDER siguen una política de Acceso Abierto y se respaldan en una Licencia CreativeCommons Atribución-NoComercial 4.0 Internacional.

\section{Revista de Estudios} Latinoamericanos sobre Reducción del Riesgo de Desastres (REDER)

Diseño: Lupe Bezzina Tipografía: Hospital 


\section{ORGANIZACIÓN DE LA GESTIÓN INTEGRAL DEL RIESGO DE DESASTRES EN COSTA RICA Y CENTROAMÉRICA.}

Costa Rica cuenta con instrumentos importantes en el tema de la Gestión Integral del Riesgo: legislación, planificación y un sistema de organización articulado que enlaza sectores institucionales y comunidades que han permitido abarcar todo el país y disminuir el riego de desastre; sin embargo, aún no se ha insertado la temática de patrimonio cultural dentro de esta organización.

La atención de la última emergencia ocasionada por el Huracán Otto en noviembre de 2016, que azotó la región centroamericana, ingresando por territorio nicaragüense e impactando territorio costarricense con una intensidad categoría 2, con vientos de hasta $175 \mathrm{~km} / \mathrm{h}$, permitió visibilizar las fortalezas y debilidades del Sistema Nacional de Gestión de Riesgos de Costa Rica: un sistema articulado que permitió abarcar todo el país con diferentes grados de alerta, con su base de operaciones en la capital, en comunicación directa con los comités locales en cada cantón del país. Pero también se evidenció que aún queda mucho trabajo por hacer en el tema de la prevención.

Su impacto afectó a las comunidades de la Zona Norte, Upala, Bagaces y Bajagua, principalmente, causando daños materiales de gran envergadura en edificaciones, infraestructura vial, eléctrica y de telecomunicaciones y en áreas naturales, con la lamentable pérdida de 10 vidas humanas.

Desde los primeros informes de la formación del evento en el mar atlántico, la coordinación ininterrumpida entre el Centro Nacional de Huracanes de Estados Unidos y la Comisión Nacional de Prevención del Riesgo y Atención de Emergencia CNE, activó el Sistema Nacional de Gestión de Riesgos de Costa Rica y para el momento de tocar tierra, casi en todo el país se habían tomado acciones que van desde la preparación de albergues y suministros, hasta evacuaciones masivas.

La alerta roja inició en 18 cantones del litoral atlántico y para la hora de la entrada a tierra, más de 50 cantones, de los 81 del país, activaron los comités cantonales de emergencia ${ }^{2}$ como prevención a imprevistos en el recorrido por tierra.

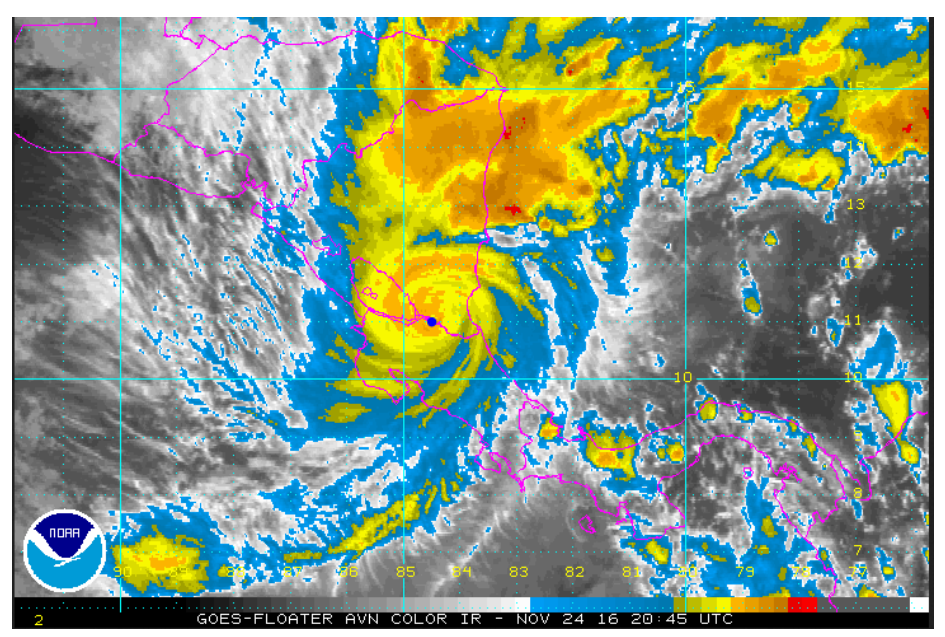

Figura 1. Imagen de satélite del momento en que el ojo del huracán ingresa al distrito de los Chiles en el territorio costarricense, luego de tocar tierra en Nicaragua. Fuente: Instituto Meteorológico Nacional de Costa Rica (IMNCR).

La activación de los comités cantonales de emergencia, constituidos en los 81 cantones del país, formados por las representaciones de instituciones púbicas como la Fuerza Pública, El Ministerio de Salud, El Cuerpo de Bomberos, La Cruz Roja, así como las Municipalidades y personas de la comunidad de cada cantón, buscan la preparación de las comunidades ante las alertas emitidas por el monitoreo de la Comisión Nacional de Prevención del Riesgo y Atención de Emergencia (CNE), líder de la Gestión Integral del Riesgo de desastres del país.

El trabajo local de los comités cantonales contribuyó a salvar cientos de vidas humanas, animales domésticos y ganado, los cuales fueron evacuados a tiempo.

Las figuras a continuación muestran las condiciones de alerta extensiva que se hizo en todo el territorio nacional; desde la prevención aun en cantones en donde no se esperaba afectación alguna, las actividades de preparación y respuesta en los cantones que serían directamente afectados hasta la atención y las primeras acciones de reconstrucción en los cantones de mayor afectación posterior al evento.
1. Otto fue el decimosexto ciclón tropical, decimoquinta tormenta, sétimo huracán de la temporada de Huracanes del Atlántico y el primero en tocar tierra costarricense. Información general en Wikipedia (2016)

2. Instancias permanentes de

coordinación en los niveles regional, Municipal y comunal. Por medio de ellos, la Comisión cumple su función de coordinación de las instituciones públicas, privadas, organismos no gubernamentales y la sociedad civil, que trabajan en la atención de emergencias o desastres. Se integran con la representación institucional o sectorial de los funcionarios con mayor autoridad en el nivel correspondiente. Las organizaciones no gubernamentales, las privadas, las locales y comunales definen su representación por medio de la autoridad interna de cada una de ellas. 


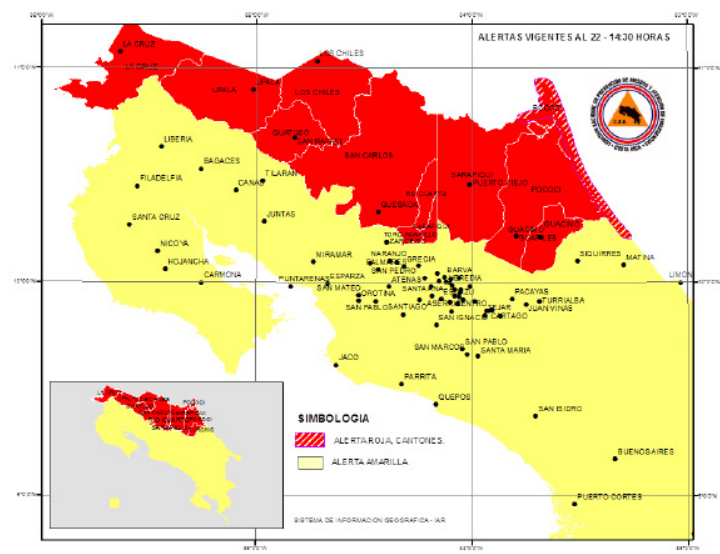

Figura a. Primeros 18 cantones en alerta roja. Fuente: CrHoy (2016).

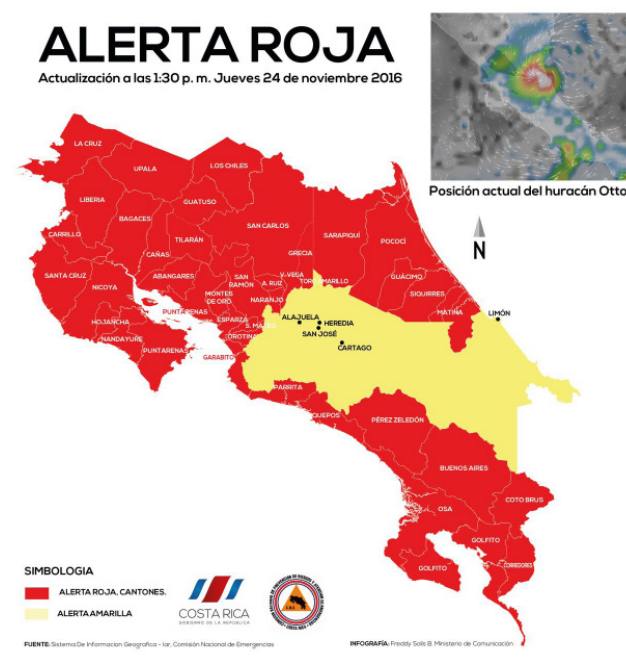

Figura c. Alerta roja en mayoría de los cantones del país a pocas horas del impacto. Ampliación a la provincia de Guanacaste. Sistema de Información Geográfica-CNE. Fuente: La Prensa Libre CR (2016).

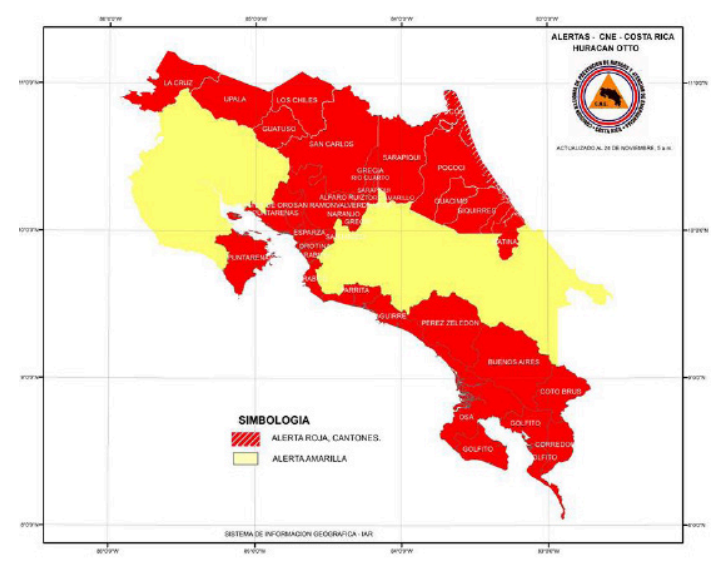

Figura b. Ampliación de la alerta a cantones del litoral pacífico. Fuente: El Mundo CR (2016).

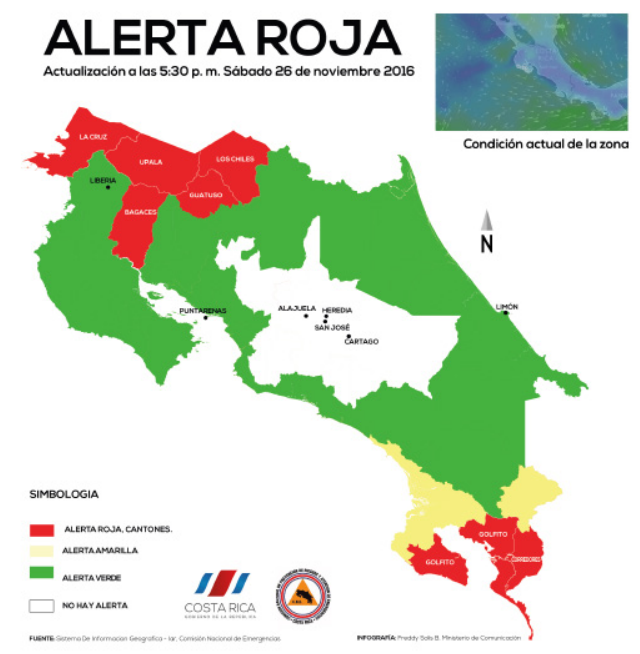

Figura d. Cantones en las que permanece la alerta luego de luego de 2 días del paso del huracán por el territorio. Fuente: La Presidencia de Costa Rica (2016).

Sin embargo, la condición de alerta roja no fue acatada con la misma seriedad en todos comités comunales de emergencia; un giro en la dirección del huracán tomó por sorpresa a comunidades que llegaron a ser las más afectadas, y donde no se dio la evacuación a tiempo con consecuencia de 10 víctimas fatales. Un registro relativamente bajo para contabilizar 23 comunidades aisladas, 412 poblados afectados y 3.655 evacuados. Sin embargo, esas muertes pudieron haberse evitado.

La organización actual en el tema de gestión de riesgo de desastres está precedida de un marco legal que ha venido actualizándose e incorporando la coordinación con lineamientos internacionales y regionales que han dado mayor solidez, recursos humanos y económicos, logrando una significativa reducción del riesgo en general; sin embargo, las amenazas son cada vez más frecuentes e intensas a causa por un lado del efecto del Cambio Climático, y por otro, de la acciones humanas como deforestación y crecimiento descontrolado en los territorios.

Actualmente la CNE cuenta con La Ley de Emergencias y Prevención del Riesgo 8488 de 2006, El Plan Nacional para la Gestión del Riesgo 2010-2015 y últimamente, la Política Nacional de Gestión del Riesgo 2016-2030 elaborada en 2015, y es en esta última en que se incorpora en el lineamiento 17 lo siguiente:

"Inversión en poblaciones vulnerables y patrimonio cultural: las instituciones públicas responsables de planificar la inversión pública en general, deben identificar y caracterizar las zonas con poblaciones vulnerables a desastres en el país, orientando las obras y la inversión social de esos lugares bajo criterios de resiliencia, que favorezcan la renovación, la adaptación, la transformación y la adopción de prácticas innovadoras en la actividad productiva, el desarrollo de asentamientos y la protección ambiental. Del mismo modo, adquiere relevancia la inversión destinada a proteger y restaurar las obras 
que son parte del patrimonio cultural del país, para las cuales cabe la posibilidad de desarrollar proyectos específicos de reforzamiento estructural, medidas especiales de protección y de aseguramiento con el fin de garantizar su longevidad. Este lineamiento tiene particular relevancia en los procesos de recuperación ante desastre".

(CNE, 2015, p.51)

En palabras del presidente de la CNE, Iván Brenes Reyes, en la presentación de la política expresa que ésta responde a los compromisos de la presente Administración presidencial como son:

"El respeto a los derechos humanos con principal atención a los grupos más vulnerables, personas con discapacidad, adulto mayor y grupos indígenas. También en materia de adaptación al cambio climático y gestión del riesgo, presentes en el Plan Nacional de Desarrollo 2015-2018, por los cuales el país aspira a que las instituciones públicas presupuesten y planifiquen la gestión del riesgo y de esta manera avanzar hacia un proceso preventivo para lograr la reducción de las pérdidas por desastre".

(CNE, 2015, p.iii)

Instituciones vitales como el ICE (Instituto Costarricense de Electricidad), el MOPT (Ministerio de Obras públicas y Transporte) y el CONAVI (Consejo nacional de Vialidad), iniciaron labores de rehabilitación inmediatamente pasaron los efectos del huracán.

Otras instituciones como el Ministerio de Educación Pública (MEP), realizó una evaluación de las instituciones educativas afectadas, asimismo el Ministerio de Vivienda realizó la evaluación de edificios junto a voluntarios del Colegio Federado de Ingenieros y Arquitectos de Costa Rica.

Si bien el mandato presidencial se cumple en la mayoría de las instituciones públicas y gobiernos locales, las oficinas del Ministerio de Cultura encargadas de la salvaguarda y conservación del patrimonio cultural, no cuentan con protocolos ni presupuestos específicos ni están integrados activamente en los comités nacionales ni cantonales de emergencia.

En Centroamérica, el Centro de Coordinación para la Prevención de Desastres Naturales de América Central, CEPREDENAC, es el organismo regional del Sistema de Integración Centroamericana (SICA) que "sistematiza y registra la información relacionada con la prevención, mitigación, respuesta, impacto y recuperación de desastres, en forma dinámica, interactiva y accesible, a nivel regional" (CEPREDENAC, 2016) demás de promover y coordinar la cooperación internacional, intercambios, asesoría técnica y científica para lograr sus objetivos para la región.

Se cuenta con la Política Centroamericana de Gestión Integral de Riesgo de Desastres (PCGIR), instrumento que regula el accionar de la institución que ha promovido la instauración de Sistemas Nacionales de Prevención, Mitigación y Atención de desastres por medio de la educación y el fortalecimiento institucional. Sin embargo, no existe una línea de trabajo para la reducción del riesgo en el patrimonio cultural y natural que se esté implementando realmente.

Las instituciones a cargo de la gestión del riesgo en cada país centroamericano son responsables de incorporar en su normativa nacional e implementar las líneas de acción en cada temática, las cuales no se analizan en este artículo.

\section{AMENAZAS AL PATRIMONIO CULTURAL ${ }^{3}$ DE LA REGIÓN CENTROAMERICANA.}

Si bien cada país del área centroamericana cuenta con amenazas específicas, en su mayoría las amenazas a las que se ha enfrentado incluyen eventos como guerras, erupciones volcánicas, sismos, desbordamiento de ríos, deslizamientos, etc. Algunos de ellos inevitables, pero en su mayoría con causas antrópicas que podrían evitarse.

Por la situación geográfica y su conformación hídrica, las amenazas más frecuentes son las inundaciones y los deslizamientos, que se incrementan con las altas precipitaciones pero que tienen mayor afectación por la deforestación y las obstrucciones de ríos y quebradas causadas por la basura depositada en sus cauces.

La ciudad de Tegucigalpa, capital de Honduras, sufrió en el año 1998 el paso del Huracán Mitch que arrasó la ciudad capital causando más de 5.600 personas fallecidas y más de 8.000 desaparecidas, provocando también grandes pérdidas en su patrimonio edificado.
Entiéndase por patrimonio cultural es el conjunto de bienes y expresiones culturales que hemos recibido de nuestros antepasados y que atestiguan las relaciones de los seres humanos. Abarca el territorio del país y la historia que se desarrolló en él, acumulada en forma de leyendas, tecnologías, conocimientos, creencias, arte y sistemas de producción y organización social (Centro de Investigación y Conservación del Patrimonio Cultural, 2017) 


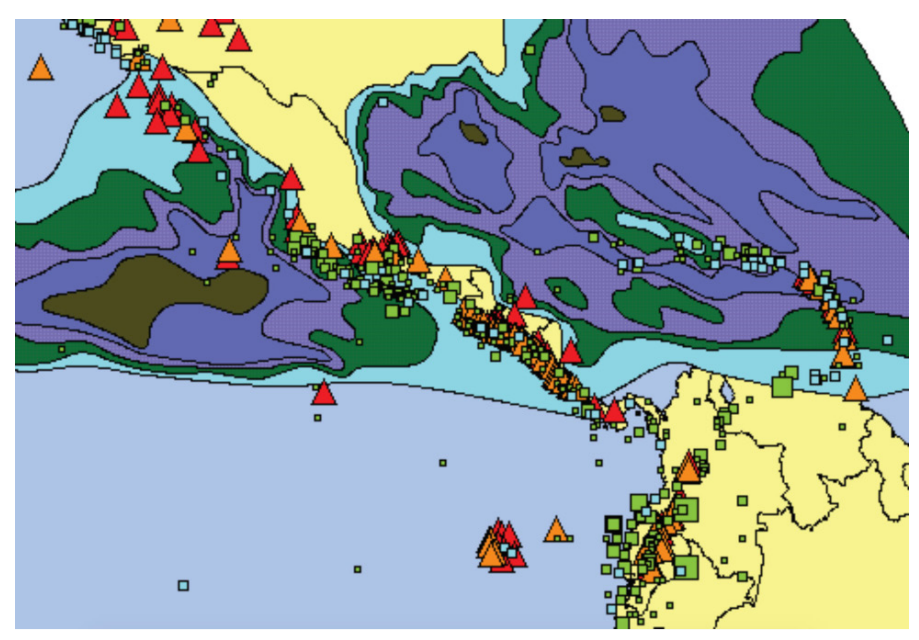

Volcánica Sísimica Tormentas tropicales

Figura 3. Tipificación de amenazas en Centroamérica. Fuente: UNISDR \& CEPREDENAC (2014).

Por su geología, la mayoría de los países del área tienen una alta actividad volcánica y sísmica que han provocado daños documentados a lo largo de su historia como el traslado de la población de Santiago de Los Caballeros (la Antigua Guatemala) a la nueva ciudad de Guatemala de la Asunción en el año 1776 y el terremoto de 1976, así como la destrucción de la ciudad de Managua en Nicaragua, arrasada durante el terremoto de 1972.

El patrimonio cultural está en riesgo latente, no solo ante eventos físicos puntuales, naturales o antrópicos, sino por factores como el desinterés del Estado e instituciones públicas que llevan a la falta de políticas públicas para su conservación, la escasa planificación en su ordenamiento territorial y a la ignorancia de su valor cultural, que aumentan su vulnerabilidad y permiten su destrucción.

La mayoría de los países centroamericanos cuentan con mapas de riesgo en donde se ubican las zonas vulnerables según tipo de amenaza. Se cuenta con evaluaciones de zonas de alta, media y baja sismicidad, la vulnerabilidad está identificada y la alerta de la creciente frecuencia e intensidad de los fenómenos naturales ante el cambio climático está presente.

Aun así, no existen levantamientos del patrimonio cultural en riesgo. Los inventarios existentes del patrimonio edificado se limitan a las edificaciones protegidas por ley, que son muy pocas, pero en su gran mayoría no se han extrapolado a los mapas de riesgo para identificar su vulnerabilidad física.

Conocer los riesgos a que está expuesto cada tipo de patrimonio da la posibilidad de crear o fortalecer estrategias de conservación, materiales o inmateriales.

La restauración de edificaciones patrimoniales se da en los casos en que ya se está a punto del colapso, traduciéndose en intervenciones agresivas y de alto costo económico.

La intervención de edificaciones patrimoniales debe iniciar antes de que sea necesaria su restauración: acciones de conservación preventiva, evaluaciones de su estado estructural para colocar los refuerzos necesarios previos a daños mayores, la evaluación de las instalaciones eléctricas cuyas fallas son consideradas como la mayor causa de incendios, apuntalamientos de estructuras en espera de una consolidación estructural, planes de mantenimiento anual ante humedades y filtraciones que dañan las estructuras, y otra gran cantidad de causas específicas con efectos que aumentan la vulnerabilidad ante eventos específicos como sismos, huracanes o sencillamente el paso del tiempo.

En cuanto al patrimonio inmaterial, la falta de respeto a las creencias y del reconocimiento de saberes tradicionales es una de las mayores amenazas. Cuando en situaciones de emergencia se debe reubicar poblaciones, el estudio de las formas de vida de esas poblaciones es vital para evitar traslados fuera de su contexto cultural.

Conocer los valores que diferencian nuestras culturas e identidades es el primer paso para enfrentar las amenazas que deterioran el patrimonio cultural de un sitio. 


\section{MANDATOS PARA LA PROTECCIÓN DEL PATRIMONIO CULTURAL Y SU INTEGRACIÓN A LA GESTIÓN INTEGRAL DEL RIESGO.}

Existe legislación nacional e internacional que establece el deber del estado y la ciudadanía de conservar su patrimonio para futuras generaciones.

Desde la constitución política de cada país, hasta normativas internacionales generales que incorporan la conservación del patrimonio como un derecho humano fundamental, hasta normas específicas que comprometen a los gobiernos a proteger el patrimonio como son:

- Convención para la Protección de los Bienes Culturales en caso de Conflicto Armado y Reglamento para la aplicación de la Convención 1954. La Haya, 14 de mayo de 1954, segundo protocolo La Haya, 26 de marzo de 1999.

- Recomendación sobre la Conservación de los Bienes Culturales que la Ejecución de Obras Públicas o Privadas pueda poner en Peligro. 19 de noviembre de 1968.

- Convención sobre las Medidas que Deben Adoptarse para Prohibir e Impedir la Importación, la Exportación y la Transferencia de Propiedad llícitas de Bienes Culturales 1970. París, 14 de noviembre de 1970.

- Convención sobre la protección del patrimonio mundial, cultural y natural. La Conferencia General de la Organización de las Naciones Unidas para la Educación, la Ciencia y la Cultura, en su 17a, reunión celebrada en París del 17 de octubre al 21 de noviembre de 1972.

- Convención para la salvaguardia del patrimonio cultural inmaterial 2003. Paris, 17 de octubre de 2003.

A estas convenciones internacionales, algunas de ellas convertidas en leyes nacionales, se suman las leyes de cultura y patrimonio de los países de la región.

En materia de Gestión del Riesgo, existen marcos internacionales, regionales y nacionales que se han desarrollado en conjunto a través de conferencias y foros participativos.

En base al análisis de la aplicación del pasado Marco de Acción de Hyogo vigente del 2005 al 2015, el preámbulo del Nuevo Marco de Acción Sendai para la Reducción de Riesgo de Desastre, 2015-2030, aprobado en la Tercera Conferencia Mundial de las Naciones Unidas sobre la reducción del Riesgo de desastres celebrada en Sendai, Miyagi (Japón) en marzo de 2015, indica en el punto 5:

"Es urgente y fundamental prever el riesgo de desastres, planificar medidas y reducirlo para proteger de manera más eficaz a las personas, las comunidades y los países, sus medios de subsistencia, su salud, su patrimonio cultural, sus activos socioeconómicos y sus ecosistemas, reforzando así su resiliencia".

(UNISDR, 2015, p.10)

El Marco de Acción de Sendai para la Reducción del Riesgo de Desastre, 2015-2030 es parte de la normativa internacional vigente que por primera vez incorpora en sus prioridades de acción, explícitamente, la incorporación del patrimonio cultural tanto en la evaluación del riesgo como en la necesidad de inversión para su protección y aumento de resiliencia, y que -según la UNISDR- sea capaz de "resistir, absorber, adaptarse y recuperarse de sus efectos de manera oportuna y eficaz, lo que incluye la preservación y la restauración de sus estructuras y funciones básicas" (UNISDR, 2015, p.9).

Dentro de la prioridad 1: Comprender el riesgo de desastres, el Marco de Acción Sendai especifica en el punto 24. Inciso d):

"Evaluar, registrar, compartir y dar a conocer al público, de manera sistemática, las pérdidas causadas por desastres y comprender el impacto económico, social, sanitario, educativo y ambiental y en el patrimonio cultural, como corresponda, en el contexto de la información sobre la vulnerabilidad y el grado de exposición a amenazas referida a sucesos específicos".

(UNISDR, 2015, p.15)

Comprender la vulnerabilidad del bien cultural y su entorno se traducirá tal y como se indica en el Marco Sendai:

"Esos conocimientos se pueden aprovechar para la evaluación del riesgo previo a los desastres, para la prevención y mitigación y para la elaboración y aplicación de medidas adecuadas de preparación y respuesta eficaz para casos de desastre".

(UNISDR, 2015, p.15) 
Como prioridad 3 establece: Invertir en la reducción del riesgo de desastres para la resiliencia. Y en el punto 29, especifica:

"Las inversiones públicas y privadas para la prevención y reducción del riesgo de desastres mediante medidas estructurales y no estructurales son esenciales para aumentar la resiliencia económica, social, sanitaria y cultural de las personas, las comunidades, los países y sus bienes, así como del medio ambiente. Estos factores pueden impulsar la innovación, el crecimiento y la creación de empleo. Esas medidas son eficaces en función del costo y fundamentales para salvar vidas, prevenir y reducir las pérdidas y asegurar la recuperación y rehabilitación efectivas".

(UNISDR, 2015, p.18)

Seguidamente, delega a las instancias a nivel nacional y local lograr "Proteger o apoyar la protección de las instituciones culturales y de colección y otros lugares de interés desde el punto de vista histórico, religioso y del patrimonio cultural" (UNISDR, 2015, p.19).

Los Objetivos del Desarrollo Sostenible al 2030 de las Naciones Unidas también se constituyen en un marco de acción ligado a lograr reducir la pobreza, poblaciones más resilientes y de un menor impacto para el planeta.

Instituciones como el PNUD y UNICEF trabajan de la mano con diferentes instancias nacionales para contribuir al fortalecimiento de capacidades en la región.

La región centroamericana cuenta con el CEPREDENAC, que desde los años 90 trabaja en todos los ámbitos de la Gestión del Riesgo y apoya a las instituciones nacionales con capacitaciones y la búsqueda de recursos, se encuentra adscrita desde el 2003 al Sistema de Integración Centroamericano, SICA.

Las instituciones centroamericanas que gestionan el riesgo de desastres en la región y que cuentan con sistemas de gestión y políticas normativas son:

- Coordinadora Nacional para la Reducción de Desastres de Guatemala, CONRED.

- La Comisión permanente de Contingencias de Honduras, COPECO.

- La Dirección General de Protección Civil de El Salvador.

- Sistema Nacional para la Prevención, Mitigación y Atención de Desastres de Nicaragua SINAPRED.

- Comisión Nacional de Prevención del Riesgo y Atención de Emergencia de Costa Rica, CNE.

- Sistema Nacional de Protección Civil de Panamá, SINAPROC.

A pesar de que ahora existe la normativa que promueve la inclusión del tema patrimonio cultural en la Gestión Integral del Riesgo de Desastres, aún es necesario fortalecer las capacidades de ambos sectores para comprender la importancia de su vinculación.

\section{ACCIONES PARA LA INTEGRACIÓN DEL PATRIMONIO CULTURAL AL SISTEMA DE GES- TIÓN INTEGRAL DEL RIESGO.}

Esta relación de temáticas ha sido desarrollada por la Organización de las Naciones Unidas para la Educación, la Ciencia y la Cultura (UNESCO) y por ICOMOS Internacional en varios países desde hace ya más de 20 años (ver, por ejemplo, convenciones internacionales en sitio web UNESCO (2017a), reportes ICOMOS (2000 y 2015) y Lista de Patrimonio Mundial en peligro UNESCO (2017b), entre otros).

En colaboración con el ICCROM-UNESCO-WHC-ICOMOS, Herb Stovel publicó en 1998 el libro Preparación ante el Riesgo: Un manual para el manejo del Patrimonio Cultural traducido al español por el ICCROM, para ser utilizado en el Seminario de Formación Regional de Preparación ante el Riesgo realizado en República Dominicana en el año 2001.

Asimismo, Stovel (s/f) elaboró el Módulo 3: Gestión de la preparación ante el riesgo, parte del Programa de desarrollo de capacidades para el Caribe (CCBP), aprobado en el 2004 por el Comité del Patrimonio Mundial desarrollado en la Habana Cuba, con el apoyo de la Unidad de Proyectos Especiales del Centro de Patrimonio Mundial, la Regional de Cultura para América Latina y el Caribe de la UNESCO y las oficinas de UNESCO en Kingston y en Puerto Príncipe. En este módulo Stovel planteó el conocimiento de conceptos básicos de la gestión del riesgo para ser aplicados en la elaboración de estrategias de preparación ante riesgos. 
En el Perú se realizó en el año 2009 el Taller sobre Gestión de Desastre en Sitios de Patrimonio Mundial del Perú y periódicamente se realizan seminarios o congresos con el apoyo de las instituciones encargadas del patrimonio.

ICOMOS Internacional lanzó en 1999 su programa Patrimonio en Riesgo, emitiendo un informe general y diversos informes de países miembros, dejando claro el panorama en ese momento. Actualmente, el ICOMOS cuenta con un comité especializado, el International Scientific Commitee on Risk Preparedness (ICORP), quienes han desarrollado capacitaciones y evaluaciones del estado del patrimonio y realizan publicaciones periódicas del patrimonio en riesgo ${ }^{4}$.

La mayor parte de las acciones están relacionadas a la conservación de patrimonio nacional declarado como Patrimonio de la Humanidad y se refieren al riesgo que sufren al no contar con la gestión necesaria del sitio, que incluye aspectos como:

- Falta de medición de los impactos por la visitación generada por el turismo.

- Falta de planes de conservación integral y monitoreo.

- Escasa formación del personal a cargo.

- Protocolos de actuación ante amenazas naturales.

- Escasa investigación a partir de sus valores patrimoniales.

- Poco compromiso de los Estados.

Riesgos como la presión demográfica, las obras públicas y/o privadas que aumentan y generan nuevos riesgos como aeropuertos, represas hidroeléctricas, etc., son comunes y afectan no solo a los sitios considerados Patrimonio de la Humanidad, sino a patrimonio nacional y local, tangible e intangible, cuyo valor es menospreciado.

En Centroamérica se han desarrollado acciones que buscan llegar no solamente al Patrimonio Mundial, sino que abarquen al patrimonio cultural en general, un espectro mucho más amplio y diverso que requiere atención.

En el año 2011, el programa ACERCA de la AECID organizó, con la colaboración de la Coordinación Educativa y Cultural Centroamericana (CECC/SICA) del Sistema de Integración Centroamericano y el Instituto del Patrimonio Cultural de España, el / Seminario sobre Gestión de Riesgos y Prevención de Desastres en Materia de Patrimonio Cultural, que se realizó del 24 al 28 de octubre de dicho año en el Centro Cultural de España en El Salvador.

El seminario estuvo dirigido a representantes de las instituciones culturales de Centroamérica, Belice y República Dominicana.

El Seminario, apoyado por la CECC/SICA tuvo como objetivo la formación de profesionales en el desarrollo, gestión y puesta en marcha de herramientas de gestión de riesgos así como de estrategias de actuación para minimizar los daños ocasionados por desastres (provocados por causas naturales y/o humanas) y procurar la salvaguarda de los bienes integrantes del Patrimonio Cultural.

Su finalidad fue la elaboración de estrategias de actuación para minimizar daños en el patrimonio cultural ante un evento.

Luego de las presentaciones de la situación en cada país, por representantes de los Ministerios/ institutos de Cultura y antropología, así como otras organizaciones relacionadas como ICOMOS y Casas de Cultura, se concluyó que el sector encargado de la gestión de Riesgos estaba ausente en el Seminario, por lo que las estrategias debían estar insertas en las estrategias de los Planes regionales y nacionales de gestión Integral del riesgo ya existentes en la región.

Como resultado, se emitieron una serie de recomendaciones y se reforzó el compromiso de trabajar por introducir el tema de la conservación del Patrimonio en la gestión Integral del Riesgo de Desastres en Centroamérica, Belice y República Dominicana.

Entre las recomendaciones emitidas para la reducción del impacto del cambio climático y prevención de desastres en el patrimonio cultural (Ministerio de Educación Cultural y Deporte de España, 2012, p.23-25), están:

- Fortalecimiento institucional.

- Articulación de los marcos jurídicos nacionales y regionales.

- Identificar las amenazas y vulnerabilidades.

- Elaboración de herramientas y metodologías de actuación.

- Insertarse en las organizaciones gestoras del riesgo de cada país para aprovechar las capacidades y avances ya establecidos.

4. Heritage at Risk. World report on monuments and sites in danger en ICOMOS (2015). 
- Establecer corresponsabilidades en la gestión.

- Elaborar y actualizar inventarios nacionales.

- Sensibilizar y formar funcionarios y técnicos de las diferentes instituciones en la temática.

- Capacitar grupos ciudadanos a nivel local y regional para la protección del patrimonio cultural.

- Promover la investigación del conocimiento tradicional ante el riesgo.

- Respetar los saberes tradicionales en la actuación ante el riesgo de desastres.

- Aumentar los recursos financieros para esta área de trabajo.

- Incorporar en la educación formal, no formal e informal los temas.

- Fomentar el trabajo multidisciplinar.

En ese compartir entre los sectores de patrimonio cultural y Gestión del Riesgo, quedó evidente la necesidad de:

- Capacitación interdisciplinar: intercambiar conocimientos específicos para actuar conjuntamente.

A partir de esta necesidad, se inicia la formulación de un proyecto conjunto entre ICOMOS Costa Rica, La oficina de UNESCO San José y CEEC/SICA (2012-2013), con el objetivo de:

"Fortalecer las capacidades de las instituciones nacionales (ministerios de cultura, ministerios de educación en particular, así como otras instancias de Estado: ministerios de vivienda, ministerios de ambiente, gobiernos locales, instituciones de prevención de riesgos/emergencia, universidades, colegios profesionales de ingenieros y arquitectos, etc.) y comunidades vulnerables, para prevenir y reducir el impacto del cambio climático y el riesgo de desastre en el Patrimonio Cultural Centroamericano".

(Baker, Cárdenas \& Esquivel, 2013, p.64-74)

El proyecto plantea realizar un curso virtual con la participación de instituciones gubernamentales, académicas y organizaciones no gubernamentales, para pasar luego a seleccionar un sitio patrimonial en riesgo en donde hacer el curso presencial, aplicando los conocimientos adquiridos en el curso virtual, y replicándolo a otras comunidades con patrimonio en riesgo

En el Simposio del ADCOM 2013 de ICOMOS Internacional ${ }^{5}$, se presentó el proyecto con la ponencia Reducción del impacto del cambio climático y de riesgos y desastres en el patrimonio cultural de Centroamérica: Proyecto Regional, y fue publicado en la memoria del evento. Este proyecto se encuentra en proceso de búsqueda de fondos para su implementación.

El planteamiento del proyecto anterior, además de involucrar el intercambio de conocimientos entre las entidades a cargo de la gestión de riesgos y de la conservación del patrimonio cultural, se basa en que la educación es un factor clave para lograr incidir en la población de las comunidades para reconocer los riesgos, minimizar la vulnerabilidad y optimizar la atención del patrimonio cultural ante amenazas a las que puedan verse expuesto.

- Oficialización de la integración de los temas: Gestión de riesgo en el patrimonio cultural.

En un intento por introducir el tema, la Asociación Costarricense para la Conservación de Monumentos y Sitios, ICOMOS Costa Rica, participó en II Foro Consultivo Regional de la PCGIR, realizado en San José, Costa Rica, los días 18 y 19 de junio de 2013.

Durante la participación en la mesa del Eje Articulador B de la PCGIR, Desarrollo y Compensación Social para Reducir la Vulnerabilidad ${ }^{6}$, se solicitó la necesidad de que se incorpore el Patrimonio Cultural en las líneas de acción y en las recomendaciones del foro, porque para crear una cultura de prevención, se debe primero fortalecer la cultura identitaria de cada sitio.

Como logro de la participación en este II Foro consultivo, se incorporó en la Declaración de San José "Por el derecho a la prevención: consolidando la participación en gestión integral del riesgo en Centroamérica" el punto 14 entre 46 recomendaciones, que indica: Fortalecer la incorporación del tema del patrimonio cultural en los planes y acciones de la gestión integral del riesgo que fortalezca la cultura identitaria.

Como seguimiento a esta iniciativa, ICOMOS de Costa Rica y la Coordinación Educativa y Cultural Centroamericana del Sistema de Integración Centroamericana CEEC/SICA, realizó a partir de Julio de 2014 y por 8 semanas, el Foro Virtual de discusión: Gestión Integral de Riesgos de desastres y Adaptación al cambio Climático para la Conservación del Patrimonio Cultural y Natural en las

\footnotetext{
5. Simposio celebrado en Costa Rica en octubre del 2013.

6. Que incorpora 3 medidas prioritarias: 1) Incorporación de la gestión de riesgos en la educación formal y no formal, 2) vivienda y ordenamiento territorial con enfoque de gestión de riesgos, y 3 ) inversión en infraestructura social básica con criterios de gestión de riesgo.
} 
Américas con el apoyo de la plataforma de la Federación Internacional de Sociedades de la Cruz Roja y de la Media Luna Roja en la página web de Desaprender.org (2017).

Se contó con la participación de más de 60 personas de 14 países de América Latina, muchos de ellos profesionales de varias disciplinas, arquitectos, ingenieros, sociólogos, ambientalistas, educadores, gestores culturales y geógrafos, entre otros, ligadas a la protección del patrimonio cultural en organizaciones e instituciones nacionales y académicas, así como profesionales independientes vinculados a comunidades vulnerables frente al riesgo de desastres y el impacto del cambio climático en el patrimonio cultural.

Este foro se realizó con el fin de establecer una comunicación directa entre las instituciones, organizaciones y comunidades para conocer las experiencias exitosas, que ya se han realizado en algunos países de Sudamérica y el Caribe, así como establecer las necesidades de cada país.

Como conclusión quedó plasmada la necesidad urgente de incorporar la conservación del patrimonio cultural en las líneas de trabajo de la Gestión Integral del Riesgo, que para el año 2014 no aparecían claramente en las agendas de la región ni en los Marcos de Acción Internacional, aun cuando la problemática común que sufre el patrimonio cultural en Las Américas es un punto de enlace para la creación de redes de trabajo.

Se cumplieron los resultados esperados a través del Foro Virtual tal y como se establecieron en el programa y el informe final del foro:

- Ruta de trabajo/plan de acción en gestión integral del riesgo y adaptación al cambio climático para la conservación del patrimonio cultural y natural.

- Insumos para contribuir en la construcción del Marco Internacional Post 2015 para la RRD (HFA 2) conocido como Marco de Acción de Sendai.

Se propuso un Plan de Acción para ser enriquecido con la difusión y socialización en los diferentes ámbitos de trabajo de los participantes al foro, asumiendo el compromiso de seguir adelante para adecuarlo a las realidades específicas de cada país o región y lograr la Integración de la Conservación del patrimonio cultural y natural en las agendas de Gestión Integral del Riesgo y Adaptación al Cambio Climático en la región.

Los valiosos aportes de los participantes de diversas disciplinas y contextos sociales y laborales demuestran que con el trabajo conjunto se logran avances significativos en un menor tiempo. La socialización de los resultados entre las personas de los 14 países participantes y las organizaciones involucradas en el foro, CEEC/SICA, ICOMOS CR e UNESCO se unió a la voz de la sociedad civil y otras instancias que en todo el mundo intentaron incidir en la inclusión del tema en el Marco de Acción de Sendai.

Entre los objetivos del plan de acción se definieron procesos de sensibilización de las personas de instituciones relacionadas con la gestión de riesgos, así como de patrimonio cultural, sobre la necesidad de coordinación a lograrse mediante intercambio de conocimientos y experiencias.

INTEGRACIÓN DEL PATRIMONIO CULTURAL A LA GESTIÓN DEL RIESGO DE DESASTRES.

El Sistema Nacional de Gestión del Riesgo de Costa Rica (SNGR) se entiende como:

"La articulación integral, organizada, coordinada y armónica de los órganos, las estructuras, las relaciones funcionales, los métodos, los procedimientos y los recursos de todas las instituciones del Estado, procurando la participación de todo el sector privado y la sociedad civil organizada".

(CNE, 2015, p.21)

Este funciona como un sistema articulado que inicia desde la CNE responsable junto a la Presidencia de la República, para guiar a las instituciones locales de soporte vital como Bomberos y Cruz Roja y las instituciones públicas relacionadas a vialidad, salud, entre otras, extendiéndose a todos los gobiernos locales (municipalidades), quienes, junto a las representaciones de las instituciones públicas y las comunidades organizadas, logran reducir eficazmente los riesgos de desastres.

La Gestión Integral de Riesgo de Desastres (GIRD) se encuentra inserta en el ámbito de la educación a nivel universitaria y en educación no formal para aumentar la resiliencia de las comunidades vulnerables y es aquí en donde la inclusión del patrimonio cultural debe iniciar. 
Algunos de los pasos generales a seguir son:

- Iniciar con un proceso general de sensibilización sobre el rol que el patrimonio cultural tiene en la vida humana y la importancia de conservarlo.

- Incluir capacitaciones en ambos temas para funcionarios de los Ministerios o Institutos encargados de la cultura, para lograr identificar los bienes culturales y expresiones culturales y la forma de conservarlos.

- Elaboración de inventarios, catalogación y mapeos para conocer el estado de vulnerabilidad de los bienes y expresiones culturales.

- Establecimiento de responsables de la verificación del estado de las edificaciones en cada área.

- Diseño de protocolos de actuación ante cada amenaza.

- Priorizar los principios de conservación del patrimonio en la planificación.

La integración de la gestión de riesgos en el patrimonio cultural debe incluirse en los tres subsistemas de la GIRD. A manera de ejemplo a continuación se establecen requerimientos generales para cada uno, considerando que el patrimonio cultural integra bienes y expresiones culturales diversas, el planeamiento de su protección y conservación no debe hacerse por separado. Tal como indica Stovel (1998) en su Manual para el manejo del Patrimonio Cultural Mundial, se deben equilibrar las acciones de protección para no afectar la autenticidad e integridad de los bienes siguiendo los principios de la preparación ante el riesgo para el patrimonio cultural.

Los subsistemas establecidos en el Sistema de GIRD de Costa Rica, abarcan las tres principales etapas dejando abierta la posibilidad de integrar al patrimonio cultural en sus temas específicos.

\section{a) Reducción del Riesgo}

"Programas, proyectos y medidas de regulación que transversalicen la gestión del riesgo en la planificación y en las prácticas sociales y económicas para evitar, mitigar, transferir y retener el riesgo".

(CNE, 2017)

Prevención. Luego de identificados los riesgos se puede trabajar en minimizarlos.

\section{Patrimonio inmueble:}

- El mapeo de las edificaciones patrimoniales en las zonas de riesgo permite identificar los tipos de amenazas y las prioridades de actuación. Esta es una tarea pendiente en la región según consulta realizada a los participantes en el Foro Virtual realizado por la CEEC/ SICA e ICOMOS CR en el año 2014.

- Los mapas de riesgo sísmico existentes constituyen una herramienta que permite priorizar acciones. La valoración y reforzamiento de edificaciones con vulnerabilidades estructurales debe realizarse previo a cualquier amenaza.

- Cada vez son más extensas las zonas inundables o propensas a deslizamientos por lo que los mapeos deben actualizarse periódicamente.

- Campañas de mantenimiento preventivo para asegurar el mejor estado de los elementos como cubiertas de techo, instalaciones eléctricas, estado de puertas y ventanas, salidas de emergencia y señalizaciones, equipo de emergencia como extintores, etc.

\section{Patrimonio mueble:}

- Los museos son los principales custodios del patrimonio mueble, que en su mayoría cumplen con los protocolos necesarios para salvaguardar las colecciones con algunas limitantes.

- Gran cantidad de patrimonio mueble que se encuentra dentro o en los alrededores de edificaciones patrimoniales como iglesias, instituciones educativas, viviendas privadas, plazas y parques, etc., no se encuentran inventariados y no cuentan con responsables para su protección.

\section{Patrimonio intangible:}

- El fortalecimiento de las identidades, saberes, formas de cultivo, oficios tradicionales, celebraciones importantes, etc., deben conocerse y documentarse para su transmisión a futuras generaciones. 


\section{b) Preparativos y Respuesta para Desastre}

"La generación de las capacidades nacionales para una respuesta rápida, efectiva y coordinada ante emergencias, cuyo propósito inmediato es la protección de la vida humana y los bienes y el avance paulatino hacia una fase posterior de recuperación de las poblaciones afectadas".

(CNE, 2017)

Preparación-Actuación. Ante una inminente amenaza o durante de ella, luego de la salvaguarda de la vida humana, se debe contar con la preparación previa y con los protocolos de seguimiento para que los riesgos no se conviertan en desastres para el patrimonio cultural.

Stovel identifica esta fase como clave para la protección efectiva del patrimonio cultural. Un plan de respuesta integrado y específico para el patrimonio cultural evitará las intervenciones inadecuadas que destruyen más que el mismo evento.

\section{El patrimonio inmueble:}

Medición del impacto y su correspondiente actuación de emergencia, que bien puede ser:

- Plan de respuesta inmediata.

- Evacuaciones.

- Apuntalamiento temporal de áreas con fallas menores.

- Colocación de cubiertas temporales.

- Vigilancia de áreas abiertas.

- Monitoreo de riesgos por falla eléctrica.

Patrimonio mueble:

- Verificación del resguardo de colecciones o su efectiva movilización.

- Vigilancia y sistemas de seguridad.

- Intervenciones de bajo impacto.

\section{Patrimonio inmaterial:}

- Respeto a saberes ancestrales

\section{c) Recuperación ante Desastres}

"La planificación de las acciones de recuperación de corto, mediano y largo plazo ante desastres las acciones orientadas a la recuperación de medios de vida y la reactivación económica y social de las zonas afectadas por el desastre, desarrolladas bajo otros instrumentos de planificación, en términos tales que se reviertan las condiciones de vulnerabilidad pre existentes".

Respuesta. Intervenciones para revertir los daños, movilizaciones para atender a las comunidades afectadas.

\section{Patrimonio inmueble:}

- Evitar intervenciones inadecuadas en entornos culturales específicos, como, por ejemplo, evitar experiencias como reconstrucciones de viviendas en territorios indígenas en los cuales se implantaron modelos con sistemas constructivos y tipologías fuera de su contexto ambiental y cultural.

- Realizar intervenciones rápidas de consolidación estructural.

- Evitar perdida de autenticidad.

- Mantener criterios de intervención establecidos en cartas internacionales de patrimonio.

- Procurar la restauración de los inmuebles manteniendo su integridad.

- Realizar restauraciones con profesionales capacitados.

- Asegurar la participación de los usuarios del bien.

\section{Patrimonio Mueble:}

- Restauración por personal calificado.

- Puesta en uso de los bienes muebles.

\section{Patrimonio inmaterial:}

- Priorizar en la recuperación del contexto cultural.

- Delegar responsabilidades a líderes.

- Instaurar los oficios tradicionales. 
SUBSISTEMA DE REDUCCION DEL RIESGO

PREVENCIÓN
Gestión correctiva del riesgo
Comprensión del riesgo.
Gestión prospectiva del riesgo.
Recursos humanos y
económicos.

SUBSISTEMA DE PREPARATIVOS Y RESPUESTA PARA DESASTRE

PREPARACIÓN- ACTUACIÓN

Protocolos.

Procedimientos establecidos

Coordinación Institucional.

Descentralización.

Delegación de responsabilidades.
SUBSISTEMA DE RECUPERACIÓN ANTE DESASTRE

\section{RECONSTRUCCIÓN}

Planificación de la recuperación.

Restauración.

Mantenimiento de criterios de intervención

Figura 4. Subsistemas del Sistema Nacional de Gestión del Riesgo de Costa Rica Integrados a la Conservación del Patrimonio Cultural. Fuente: Elaboración de la autora.

\section{DESAFÍOS DE LA CONSERVACIÓN DEL PATRIMONIO CULTURAL Y NATURAL ANTE EL RIEGO DE DESASTRE.}

Lamentablemente, se concluye que a pesar de los antecedentes y lineamientos para gestionar el riesgo en el patrimonio cultural, que desde el sector cultura se han desarrollado en todo el mundo, aún no han logrado integrarse a los sistemas nacionales de gestión del riesgo de desastres.

El reconocimiento de la importancia de conservar, no solo el Patrimonio de la Humanidad declarado como tal, sino también el patrimonio cultural, tangible e intangible en general, es también una tarea pendiente para lograr incidencia en todos los ámbitos de la vida de la humanidad.

La incorporación del tema patrimonio cultural en el Marco Internacional de Acción Sendai, en la Política Centroamericana (PCGIR) y en la Política Nacional de Gestión del Riesgo 2016-2013 de Costa Rica, es un gran paso para continuar con el proceso de comprensión y reconocimiento del valor del patrimonio cultural para la vida de los pueblos.

El Marco de Sendai establece entre sus prioridades la comprensión del riesgo: conocer específicamente los riesgos que sufre el patrimonio cultural y sus implicaciones es urgente para tomar acciones para su protección. En este sentido, el proyecto de formación de capacidades interdisciplinarias formulado por UNESCO-ICOMOS CR-CEEC/SICA es necesario para iniciar la diseminación de las acciones adjuntas en toda la región.

El resguardo de la vida humana como prioridad de la Gestión del Riesgo, no puede desligarse de la salvaguarda de sus identidades y de su contexto cultural. Los desastres no pueden medirse solamente por las pérdidas materiales, de los medios de subsistencia y producción o vidas animales y humanas. La existencia de desastres intangibles producidos por no considerar el planteamiento diferenciador, que debe existir en la conservación preventiva (prevención), evaluación e intervención adecuada (actuación) y la restauración o rehabilitación (reconstrucción) de los bienes y expresiones culturales, debe detenerse.

Contar con los instrumentos legales, normativas y planes de acción conjuntos, si bien delimitan y organizan las actuaciones en cada etapa de la Gestión Integral del Riesgo, deben complementarse con formación de capacidades en ambos temas. Los gobiernos locales, en quienes finalmente descansa la responsabilidad de ejecutar las acciones, deben tener conocimiento de la localización de su patrimonio edificado, de los bienes culturales muebles y de las formas de vida y expresiones de las comunidades en su territorio, para lograr su protección.

La participación ciudadana y de organizaciones sin fines de lucro como el ICOMOS y otras similares han logrado concretar acciones que han incidido en que se incorpore el tema en las agendas de la Gestión Integral del Riesgo de Desastre a nivel mundial. Debe existir un compromiso desde el sector cultura para participar activamente en todos los procesos y lograr que se implementen las acciones en cada etapa de la GIRD.

Los esfuerzos aislados deben convertirse en estrategias nacionales y regionales para lograr avanzar en el desafío de integrar realmente la conservación del patrimonio cultural en los Sistemas de Gestión Integral del Riesgo de Desastres y la Reducción del Impacto al Cambio Climático. 


\section{REFERENCIAS}

Baker, J., Cárdenas, C. \& Esquivel, B. (2013). Reducción del Impacto del Cambio Climático y de Riesgos y Desastres en el Patrimonio Cultural de Centroamérica: Proyecto Regional. UNESCO-ONU, UNESCO SJ, CECC-SICA, ICOMOS-CR. En Avgerinou-Kolonias, S., Campos O., M., \& Barzuna P., G. (Eds.),Tangible Risks, Intangible Opportunities: Long Term Risk Preparedness and Responses for Threats to Cultural Heritage. Reducing Risks to Cultural heritage from Uncontrolled Development in a Globalized World. ICOMOS International Scientific Symposium 2013. San José, C.R: Editorial UCR.

CEPREDENAC. (2016). Acerca del CEPREDENAC. Recuperado el 16 de abril de 2017 del sitio web del CEPREDENAC : http://cepredenac.org/index.php/about/acerca-del-cepredenac

Centro de Investigación y Conservación del Patrimonio Cultural. (2017). Patrimonio Cultural. Recuperado el 3 de abril de 2017 del sitio web del Ministerio de Cultura y Juventud: http://www. patrimonio.go.cr/patrimonio/index.aspx

Comisión Nacional de Prevención de Riesgos y Atención de Emergencias (CNE). (2015). Política Nacional de Gestión del Riesgo 2016-203o. San José, C.R.: CNE.

Comisión Nacional de Prevención de Riesgos y Atención de Emergencias (CNE). (2017). El Sistema Nacional de Gestión del Riesgo. Recuperado el 15 de febrero de 2017 del sitio web CNE: http://politica.cne.go.cr

Desaprender.org. (2017). Foros. Recuperado el 20 de abril de 2017 del sitio web del Desaprender.org: http://www.desaprender.org/forum-directory

ICOMOS. (2000). Informe mundial 2000 de ICOMOS sobre monumentos y sitios en peligro. Tendencias, amenazas y riesgos. Recuperado el 7 de enero 2017 del sitio web ICOMOS: https://www. icomos.org/risk/world_report/200o/trends_spa.htm.

ICOMOS. (2015) Heritage@Risk. Recuperado el 27 de Marzo de 2016 del sitio web ICOMOS: http:// www.icomos.org/en/get-involved/inform-us/heritage-alert/heritage-at-risk-reports.

Ministerio de Educación Cultura y Deporte de España. (2012). Recomendaciones del I Seminario Sobre Gestión de Riesgos y Prevención de Desastres en Materia de Patrimonio Cultural. San Salvador (El Salvador). Patrimonio Cultural de España, Patrimonio en riesgo: seísmos y bienes culturales 6(1), 23-25.

Stovel, Herbert. (1998). Preparación ante el Riesgo: Un manual para el manejo del patrimonio cultural mundial. Traducción al español. Paris: ICCROM.

Stovel, Herbert. (s/f). Módulo 3. Gestión de la Preparación ante el riesgo. CCBP. Programa de desarrollo de capacidades para el caribe para el patrimonio mundial. La Habana: UNESCO. Recuperado el 28 de Marzo 2017 del sitio web de UNESCO: http://unesdoc.unesco.org/images/o021/oo2170/217016s.pdf

UNISDR. (2015). Marco de Sendai para la Reducción del Riesgo de Desastres 2015-2030. Ginebra: UNISDR.

UNISDR \& CEPREDENAC. (2014). Informe del Estado de Vulnerabilidad y Riesgo de Desastres en Centroamérica. Ciudad de Panamá: UNISDR.

UNESCO. (2017a). Convenciones Internacionales. Recuperado el 24 de abril de 2017 del sitio web de UNESCO: http://portal.unesco.org/es/ev.php-URL_ID=12025\&URL_DO=DO_TOPIC\&URL_ SECTION=-471.html

UNESCO. (2017b). Lista de Patrimonio Mundial en peligro. Recuperado el 16 de abril de 2017 del sitio web de UNESCO: http://whc.unesco.org/en/danger/

Wikipedia. (2016). Huracan Otto. Recuperado el 23 de Diciembre de 2016 del sitio web de Wikipedia: https://es.wikipedia.org/wiki/Hurac\%C3\%Aın_Otto\#cite_note-24.

\section{IMÁGENES DE MAPAS}

CrHoy. (2016). Primeros 18 cantones en alerta roja, mapa. Recuperado el 16 de abril de 2017 del sitio web de CRHOY: http://www.crhoy.com/nacionales/estos-son-los-18-cantones-en-alerta-roja-por-otto/

El Mundo.cr. (2016). Ampliación de la alerta a cantones del litoral pacífico, mapa, Recuperado el 26 de abril de 2017 del sitio web de El Mundo: http://cdn.elmundo.cr/wp-content/uploads/2016/11/alerta-roja-huracan-otto.jpg 
IMNCR (Instituto Meterorológico Nacional). (2016). Huracán Otto, mapa. Recuperado el 16 de abril de 2017 del sitio web de IMN: https://www.imn.ac.cr/web/imn/inicio

La Prensa Libre. Cr. (2016). Alerta roja en mayoría de los cantones del país a pocas horas del impacto. Ampliación a la provincia de Guanacaste. Sistema de Información Geográfica-CNE, mapa. Recuperado el 17 de abril de 2017 del sitio web de Prensa Libre: http://www.laprensalibre.cr/Noticias/ detalle/94283/otto-mantiene-alerta-roja-en-la-mayoria-del-territorio-nacional

La Presidencia de Costa Rica. (2016). El país amaneció con solo 7 cantones en alerta roja, mapa. Recuperado el 16 de abril de 2017 del sitio web de la Presidencia: http://presidencia.go.cr/ comunicados/2016/11/el-pais-amanecio-con-solo-7-cantones-en-alerta-roja 